\title{
Intonation-contingent adaptation to speech
}

\author{
JEFFREY L. ELMAN \\ Department of Linguistics, University of California, San Diego, La Jolla, California 92093
}

\begin{abstract}
Contingent adaptation effects have previously been reported with vowel, amplitude, ear, and duration of formant transition. The present study presents evidence that adaptation to place of articulation can be made contingent on intonation contour, as defined by pitch changes occurring during the second half of the vowel portion of the stimuli. It is argued that the most satisfactory account of this effect is that adaptation has induced changes in response bias rather than fatigue of feature detectors.
\end{abstract}

A number of recent studies have suggested the existence of feature detectors for speech; these are claimed to be discrete neurolinguistic mechanisms which extract phonetic and/or acoustic features from the speech wave. The hypothesis that these feature detectors mediate the perception of speech was advanced initially on largely theoretical grounds by Abbs and Sussman (1971). Although there has since then been little direct evidence for feature detectors, results of experiments involving the selective adaptation to speech have been interpreted as supportive of the feature detector hypothesis (Ades, 1976; Cooper, 1975; Eimas \& Corbit, 1973; Eimas \& Miller, 1978).

The selective adaptation paradigm involves comparison of subjects' labeling functions of synthetic speech stimuli before and after exposure to repetitions of an endpoint stimulus (the adaptor). Successful adaptation consists of a shift in the location of the phoneme boundary toward the adapt end of the continuum, such that fewer of the stimuli are perceived as belonging to the same category as the adaptor after adaptation compared with before. The usual explanation of this shift is that adaptation selectively fatigues the feature detector responsible for perceiving the phonetic or acoustic feature which distinguishes one end of the continuum from the other end. After adaptation, the fatigued feature detector responds more weakly than its opponent; this causes a change in the identification of stimuli near the phoneme boundary.

In an interesting variation of this paradigm, Cooper (1974) obtained what he called "contingent adaptation" effects. To obtain contingent adaptation, two sets of test stimuli are used instead of one. Both continua vary along one dimension (e.g., from a voiced to a voiceless stop in a CV syllable), while differing from each other along another dimension (e.g., the vowel of the same syllable). Two alternating stimuli are used

This work was supported by N.S.F. Grant $79-01670$ to the author. Address reprint requests to J. L. Elman, Department of Linguistics, C-008; University of California, San Diego; La Jolla, California 92093. as adaptors, the first from one end of one test set and the other from the opposite end of the second. For example, one might use test stimuli ranging from $/ \mathrm{ba} /$ to $/ \mathrm{pa} /$ and from $/ \mathrm{bi} /$ to $/ \mathrm{pi} /$; adaptors could be /ba/ alternating with $/ \mathrm{pi} /$. In such a situation, what happens is that, rather than the voiced and voiceless adaptors canceling in their effects, each test series is affected more by the adaptor from that series than by the adaptor from the other series. Thus, the phoneme boundary moves toward the voiced end of the /ba-pa/ set but toward the voiceless end of the /bi-pi/ set. In this example, the effect of voicing adaptation can be said to have been made contingent on vowel quality.

It has seemed to many that contingent adaptation provides evidence for the sensory nature of adaptation (rather than a response bias account; cf. Elman, 1979). After all, it could be argued, how can the perceptual system segregate the effects of hearing the /ba/ adaptor from the /pi/ adaptor? On the other hand, it is not at all clear why response bias should be constrained from operating at subphonemic levels. Findings reported by Diehl, Elman, and McCusker (1978) and Diehl, Lang, and Parker (in press) suggest that contrast effects may occur even when contrasting elements are only subtly related. The ability to attend to rather fine acoustic and phonetic details makes it difficult to rule out a response bias account of contingent adaptation.

Contingent adaptation has also seemed to indicate that feature detectors operate at an acoustic, rather than phonetic, level of processing (Ades, 1976). Phonetically, the consonantal portions of the $/ \mathrm{ba} /$ and the /pi/ adaptors have opposite values for the feature (voiced), and hence should cancel. However, the consonantal formant transitions (which carry the information about voicing) differ spectrally as a consequence of the following vowel. Only feature detectors which are sensitive to frequency-specific cues to voicing rather than a global (i.e., phonetic) voicing feature could be expected to be differentially affected by the $/ \mathrm{ba} /, / \mathrm{pi} /$ sequence.

It has been possible to make adaptation to either place of articulation or voicing contingent not only on 
vowel, but also amplitude (Ganong, Note 1), ear (Ades, 1974), pitch (Ades, 1977), and duration of formant transition (Dechovitz \& Mandler, Note 2). In the vast majority of cases reported to date, the dimension upon which adaptation has been made contingent has introduced serious acoustic effects into the first dimension (although, phonetically, the first dimension remains the same). The single exception is the report by Ades (1977) that adaptation by voiceless plosives (/phae/ and /thae/) on a voiced series (/bae/-/dae/) could be made contingent on the $F \emptyset$ of the following vowel. Ades interpreted this effect as demonstrating an interaction between feature extraction and source assignment, where sources are defined by the pitch, intensity, and spatial location of the stimulus. An important implication of the Ades (1977) experiment was that the source assignment of a stimulus might depend, in part, on the nature of segments adjacent to it. (The question of how large the temporal window is over which source information is integrated remains unanswered.) Ades suggested that this integration occurs during precategorical acoustic store (PAS).

There were two goals in the present study. The first was to replicate the finding that adaptation can, indeed, be made contingent on a parameter which does not affect the adapted dimension either acoustically or phonetically. The second goal was to find a contingent dimension which was associated with a relatively highlevel linguistic variable, and thus to see whether feature extraction might be at all dependent on (presumably) late-occurring cognitive processes.

To accomplish this, a set of stimuli was used which varied along the dimension of place of articulation; stimuli ranged perceptually from $/ \mathrm{ba} /$ to $/ \mathrm{da} /$. The second dimension, along which adaptation was made contingent, was intonation contour. Unlike the stimuli in Ades' (1977) study, FO was varied only over the second half of the vocalic portion of each stimulus. In this way, pairs of stimuli could be generated which sounded different (although from the same source), due to different pitch contours, but whose consonantal portions were physically identical.

A model of speech perception involving passive feature detectors would predict a failure to obtain a postadaptation phoneme boundary shift under such conditions, since the acoustic and phonetic character of the stimuli ought to cause simultaneous and equal fatigue of both the bilabial- and alveolar-sensitive detectors. These detectors ought to be insensitive to information such as the intonation contour of the following vowels, especially since these contours provide no information (such as source) about the phonetic character of the stop. On the other hand, if adaptation can be made contingent on intonation, this suggests that feature extraction is an active process in which abstract stimulus attributes which may be of no obvious intrinsic relevance may play a role.

\section{METHOD}

\section{Stimuli}

Seven stimuli were generated on an OVE IIId serial resonance synthesizer under control of a PDP-12 computer. The stimulus F2 and F3 onset frequencies are displayed in Table 1. Transitions lasted $40 \mathrm{msec}$, after which steady-state values appropriate to the vowel /a/ were maintained for the remaining $240 \mathrm{msec}$ of each syllable. Stimulus 1 was heard as a clear $/ \mathrm{ba} /$, stimulus 7 as a clear $/ \mathrm{da} /$; but the intermediate stimuli were constructed so as to cluster close to the perceived phoneme boundary.

Two sets of stimuli were created, differing only in their pitch contours (schematic versions of typical stimuli are displayed in Figure 1). The fundamental in both sets rose from 79 to $119 \mathrm{~Hz}$ within the first $10 \mathrm{msec}$ after release; this value was maintained through the first half of all the stimuli (and, importantly, well past the consonantal transitions). After $140 \mathrm{msec}, \mathrm{FO}$ rose exponentially in the first set to $308 \mathrm{~Hz}$, and fell in the second set to $50 \mathrm{~Hz}$. The stimuli were perceived as having contours appropriate to exaggerated questions or declarative statements. (These two sets of stimuli will be referred to henceforth as /Rba-Rda/ and /Fba-Fda/, respectively.) Importantly, all stimuli were perceived as originating from the same source (spatial location and vocal tract).

Ten copies of each of the 14 stimuli were recorded with an Ampex AG500 recorder on audio tape. Stimuli were ordered such that blocks of seven rising $F \boldsymbol{F}$ stimuli alternated with blocks of seven falling $F \emptyset$ stimuli, with stimuli ordered randomly within blocks. This tape was used as the preadaptation identification test tape. Two adaptation tapes were constructed in the following manner. One of the tapes contained an initial period of $1.5 \mathrm{~min}$ during which the sequence Rba Fda Rba Fda . . . was recorded; on the other tape, the sequence was Rda Fba Rda Fba . . . . The four stimuli used were taken from the endpoints of the two test continua. Interadaptor interval (IAI) was $150 \mathrm{msec}$. [The exact structure of the contingent adaptation sequence, as well as the IAI, may be very important. Pilot work had revealed that some other adaptation sequences and IAIs resulted in the perception of polysyllabic words, e.g., bada bada (cf. also Hall \& Blumstein, 1978), rather than as a sequence of alternating monosyllables. This was probably due to the role of pitch contour in cuing the perception of stress placement.] Following the initial adaptation, a single test item was recorded. From this point on, 30 alternations of the adaptation pairs preceded every test item, using the same order as the preadaptation test tape. In addition, within each tape, the order of each member of the adaptation pair was randomly varied, so that on one of the tapes, for example, Rba Fda . . occurred before some stimuli, and Fda Rba . . . occurred before others.

\section{Subjects}

Ten undergraduates from the University of California, San Diego

Table 1

Onset Frequencies (in Hertz) of F2 and F3

\begin{tabular}{ccc}
\hline Stimulus & F2 & F3 \\
\hline 1 & 898 & 2,263 \\
2 & 979 & 2,397 \\
3 & 1,068 & 2,540 \\
4 & 1,165 & 2,691 \\
5 & 1,270 & 2,851 \\
6 & 1,385 & 3,020 \\
7 & 1,467 & 3,200 \\
\hline
\end{tabular}



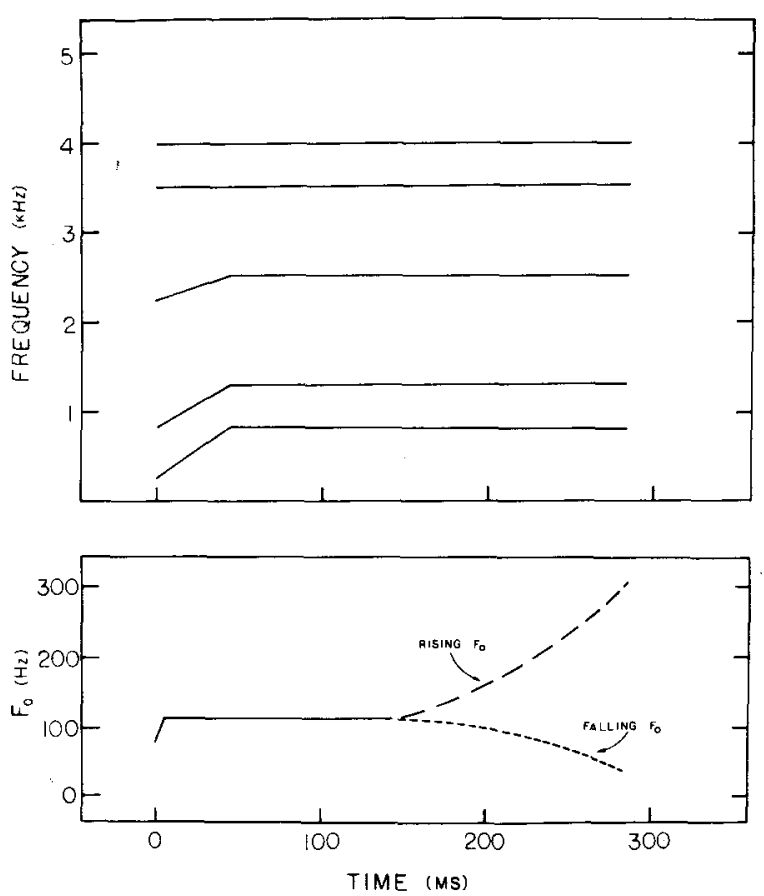

Figure 1. Schematic representation of typical stimulus pair. Top panel illustrates formant trajectory common to both rising stimulus 1 and falling stimulus 1 . Lower panel shows F0 contours. Solid line contour is shared by both $R$ and $F$ stimuli, $R$ contour is shown by long broken line, and $F$ contour is shown by short broken line.

served as subjects and received $\$ 4$ for their participation. The subjects had normal speech/hearing and were native, monolingual English speakers. One subject failed to complete the study; data are reported here for the remaining nine subjects.

\section{Procedure}

The experiment was carried out in two sessions over a period of 4 days. During the first session, the subjects were familiarized with the stimuli. The nature of the test continua was explained to them, and they listened to practice tapes until they reported being able to identify most of the stimuli with reasonable confidence. Because of the nature of the stimuli, there was some test items which remained difficult for all subjects to identify throughout the experiment.

The subjects then identified the 140 stimuli on the test tape, writing " $B$ " or " $D$ " on a prepared response sheet. After a brief rest period, the subjects listened to and identified the stimuli on one of the two adaptation tapes. The second session was similar to the first, except that the subjects heard the other adaptation tape. The order of presentation of the two adaptation tapes was counterbalanced across subjects, with half hearing one order, and half hearing the other.

\section{RESULTS}

The least mean squares method was used to calculate the phoneme boundary for every subject on each of the four tests (two preadaptation, two postadaptation). For each session, the postadaptation shift in the phoneme boundary on the rising $F \emptyset$ series was compared with the boundary shift on the falling $F \emptyset$ series and a difference score was extracted. This difference score was an indication of the relative effect of the particular adaptation sequence on the two test sets. A failure to adapt, or an equivalent effect of an adaptor on both test series, would result in a difference score of 0 . The difference scores obtained are reported in Table 2. No order effects were apparent, so the data have been collapsed across orders.

As is evident, the adaptation sequence Rba-Fda and the adaptation sequence $R$ da-Fba each affected the $\mathrm{R}$ and $\mathrm{F}$ stimuli in different ways. Rba-Fda adaptation caused a larger phoneme boundary shift toward the $/ \mathrm{ba} /$ end of the $\mathrm{R} F \emptyset$ series than on the $F \mathrm{~F} \emptyset$ series. This difference of -.34 stimulus units was significant at the level of $p<.05[t(8)=2.38$, two-tailed test]. The Rda-Fba adaptor had the opposite effect: the phoneme boundary on the $R \mathbf{F} \emptyset$ series moved +.37 units toward the $/ \mathrm{da} /$ end than on the $\mathrm{F} F$ series. This is significant at the level of $p<.01[t(8)=3.48]$. A $t$ test for correlated means reveals that the overall effect is significant at the $\mathrm{p}<.001$ level $[\mathrm{t}(8)=5.65]$.

\section{DISCUSSION}

These findings are noteworthy in that they demonstrate the ability to obtain contingent adaptation effects even when there is no plausible reason to believe that separate sets of feature detectors are being fatigued. Unlike the majority of earlier experiments, the correlated second dimension has no acoustical effect on the consonantal part of the stimuli or even on a generous portion of the vowel. The first $140 \mathrm{msec}$ of each R FV are acoustically identical to the beginning of each corresponding $F F \emptyset$ stimulus. Similarly, the Rba adaptor is opposed to the Fda adaptor only with regard to onset frequencies of F2 and F3; there is no other way in which they differ spectrally. One might thus have expected the two adaptors to have had identical effects on both sets of test stimuli and, furthermore, that those effects would cancel.

Table 2

Difference Scores for Pre- and Postadaptation R F0 and F F0 Stimuli Identifications

\begin{tabular}{lccc}
\hline \multicolumn{4}{c}{ Adapting Pair } \\
\cline { 2 - 3 } Subject & Rba Fda & Rda Fba & Difference* \\
\hline K.F. & -.96 & -.07 & +.89 \\
A.I. & +.32 & +.91 & +.59 \\
J.E. & -.25 & +.42 & +.67 \\
M.C. & -.38 & +.38 & +.76 \\
S.C. & -.01 & +.19 & +.20 \\
P.M. & -.35 & +.60 & +.95 \\
C.R. & -.79 & +.63 & +1.42 \\
D.W. & -.32 & -.02 & +.30 \\
N.N. & -.34 & +.32 & +.66 \\
Mean & -.34 & +.37 & +.72 \\
p $<$ & .05 & .01 & .001 \\
\hline
\end{tabular}

*Difference between adaptors. 
In the one previous experiment in which feature extraction was demonstrated to be carried out independently for spectrally and phonetically identical segments, the stimuli were perceived as having originated from different sources (i.e., vocal tracts). Since there is significant variation across speakers in vocal tract length and shape, it is reasonable that this variation should be taken into account when determining phonetic character and be as important as, say, vowel context, amplitude, or rate. All these variables interact importantly with phonetic features. It is therefore not at all implausible that if neural mechanisms have evolved for phonetic feature detection, they should be sensitive to the above factors. As Ades (1977) suggests, segments are probably held in PAS during the period that the factors are evaluated and phonetic features extracted.

Falling and rising intonation, on the other hand, are associated with syntactic and semantic characteristics of an utterance. There is no obvious way in which the intonation contour of a vowel supplies information regarding the phonetic nature of the preceding plosive. There is no clear reason why feature detectors should be sensitive to intonation contour in the same way that they might be sensitive to source. To suggest that feature detectors that extract place of articulation in questions are different from those that extract place of articulation in declarative statements seems highly implausible; at the very least, it opens the door to an undesirable proliferation of feature detectors.

Instead, these results suggest that feature extraction is a more active and complex process than the usual model of neural feature detectors implies. The process seems to be sufficiently flexible to make reference to a large number of stimulus attributes, not all of which are available during the limited time the stimulus is held in PAS. The particular attributes which are deemed relevant to the categorization process may depend, in part (if not in toto), on the specific listening situation. Thus, it might be possible to create a large number of ad hoc continua and manipulate subjects' category formation through selective adaptation. Remez (1979) has shown that this can be done for a synthetic speech/nonspeech continuum, and he concluded that "selective adaptation does not depend on the existence of feature detectors."

What then is the mechanism of selective adaptation? One possibility is that it involves something akin to the criterion shift associated with response bias in signal detection theory (Green \& Swets, 1974). Rather than fatiguing neural detectors at a sensory level, adaptation might cause listeners to redefine their categories and adopt stricter criteria for what constitutes membership in the adapted category. The basis for this change would appear to be the contrast between the adaptor and stimuli near the category boundary. There is evidence that a contrast effect can bring about such changes in stimulus perception not only for nonspeech continua (Appley, 1971), but, more recently, for speech stimuli as well (Diehl et al., 1978; Diehl et al., in press).

At the same time, there are a number of ways in which this mechanism is strikingly different from the way in which changes in response bias are induced. Typically, one thinks of response bias as occurring sufficiently late in the process of perception that it can be manipulated more or less consciously by observers. For example, an experimenter may persuade subjects to increase the number of false alarms they report by placing a penalty on misses. Moreover, the subjects can usually be made aware of their response biases. In contrast, subjects do not seem to be amenable to overt manipulation of their response biases on phoneme identification tests (Diehl, Note 3).

It might also seem implausible that response bias should be affected by a contrast effect under circumstances where the dimension along which the adaptor contrasts is not superficially obvious to the subject. This is typically the case in cross-series adaptation, where the adaptor shares only some characteristics with the test series (e.g., place or manner of articulation). This last objection to a response bias account of selective adaptation may not be serious. Diehl et al. (1978), using a paradigm which was designed to explicitly test for contrast effects, found that such effects could be created with cross-series stimuli. Thus, if contrast effects result in changes in response bias, it appears that responses can be biased even when subjects might not be consciously aware of the nature of the contrast.

In summary, the current results seem to indicate that adaptation can occur at an intermediate level of processing. This level lies beyond the point where the spectral and source characteristics of a stimulus are extracted; it is also not a level which plausibly involves feature detectors. It is not clear that response bias is the best term to apply to this effect; it would certainly be necessary to extend what is currently understood by this term if it is used here. In any event, until the locus and mechanism of selective adaptation can be more confidently determined, it would seem prudent to refrain from the overly specific neurophysiological modeling of speech perception that is implied by the term "feature detector."

\section{REFERENCE NOTES}

1. Ganong, W. F. Amplitude contingent selective adaptation to speech. Paper presented at the 91st Meeting of the Acoustical Society of America, Washington, D.C. April 1976.

2. Dechovitz, D., \& Mandler, R. Duration-contingent effects in adaptation. Haskins Laboratories: Status Reports on Speech Research, SR-55/56, 1978.

3. Diehl, R.L. Personal communication, 1978. 


\section{REFERENCES}

AbBs, J. H., \& Sussman, H. M. Neurophysiological feature detectors and speech perception: A discussion of theoretical implications. Journal of Speech and Hearing Research, 1971, 14, 23-26.

Aves, A. E. Bilateral component in speech perception? Journal of the Acoustical Society of America, 1974, 56, 610-616.

Aves, A. E. Adapting the property detectors for speech perception. In R. J. Wales \& E. Walker (Eds.), New approaches to language mechanisms. Amsterdam: North-Holland, 1976.

AdEs, A. E. Source assignment and feature extraction. Journal of Experimental Psychology: Human Perception and Performance, 1977, 3, 673-685.

Appley, M. H. (Ed.). Adaptation level theory. New York: Academic Press, 1971.

COOPER, W. E. Contingent feature analysis in speech perception. Perception \& Psychophysics, 1974, 16, 201-204.

COOPE R, W. E. Selective adaptation to speech. In F. Restle, R. M. Shiffrin, N. J. Castellan, H. R. Lindaman, \& D. B. Pisoni (Eds.), Cognitive theory (Vol. I). Hillsdale, N.J: Erlbaum, 1975.

Diehl, R. L., Elman, J. L., \& McCusker, S. B. Context effects in phoneme identification. Journal of Experimental Psychology: Human Perception and Performance, 1978, 4, 599-609.

Diehl, R. L., LANG, M., \& Parker, E. M. A further parallel between selective adaptation and contrast. Journal of Experimental Psychology: Human Perception and Performance, in press.

Eimas, P. D., \& Corbit, J. D. Selective adaptation of linguistic feature detectors. Cognitive Psychology, 1973, 4, 99-109.

Eimas, P. D., \& Millek, J. L. Effects of selective adaptation on the perception of speech and visual patterns: Evidence for feature detectors. In H. Pick \& R. Walk (Eds.), Perception and experience. New York: Plenum, 1978.

Elman, J. L. Perceptual origins of the phoneme boundary effect and selective adaptation to speech: A signal detection theory analysis. Journal of the Acoustical Society of America, 1979 , 65, 190-207.

Green, D. M., \& Swets, J. A. Signal detection theory and psychophysics. Huntington, N.Y: Krieger, 1974 (reprinting of 1966 edition).

Hall, L. L., \& Blumstein, S. E. The effect of syllabic stress and syllable organization on the identification of speech sounds. Perception \& Psychophysics, 1978, 24, 137-144.

REMEz, R. E. Adaptation of the category boundary between speech and non-speech: A case against feature detectors. Cognitive Psychology, 1979, 11, 38-57.

(Received for publication September 17, 1979; revision accepted December 31, 1979.) 